\title{
A mixed integer programming approach for multi-action planning for threat management
}

\author{
José Salgado-Rojas ${ }^{\mathrm{a}, 1}$, Eduardo Álvarez-Miranda ${ }^{\mathrm{b}}$, Virgilio Hermoso $^{\mathrm{c}}$, Jordi Garcia-Gonzalo ${ }^{\mathrm{c}}$, Andrés Weintraub $^{\mathrm{d}}$ \\ ${ }^{a}$ Department of Statistics and Operations Research, Polytechnic University of Catalonia, Barcelona, Spain \\ ${ }^{b}$ Department of Industrial Engineering, Faculty of Engineering, Universidad de Talca, Curicó, Chile \\ ${ }^{c}$ Forest Sciences Centre of Catalonia, Solsona, Spain \\ ${ }^{d}$ Department of Industrial Engineering, Universidad de Chile, Santiago, Chile
}

\begin{abstract}
Planning for management actions that address threats to biodiversity is important for securing its long term persistence. However, systematic conservation planning (SCP) has traditionally overlooked this aspect and just focused on identiying priority areas without any recommendation on actions needed. This paper develops a mixed integer mathematical programming (MIP) approach for the multi-action management planning problem (MAMP), where the goal is to find an optimal combination of management actions that abate threats, in an efficent way while accounting for connectiivty. An extended version of the MAMP model (MAMP-E) is also proposed that adds an expression for minimizing fragmentation between different actions. To evaluate the efficiency of the two models, they were applied to a case study corresponding to a large area of the Mitchell River in Northern Asutralia, where 45 species of freshwater fish are exposed to the presence of four threats. The evaluation compares our exact MIP approach with the conservation planning software Marxan and the heuristic approach developed in Cattarino et al. (2015). The results obtained show that our MIP models have three advantages over their heuristic counterparts: shorter execution times, higher solutions quality, and a solution quality guarantee. Hence, the proposed MIP methodology provides a more effective framework for addressing the multi-action conservation problem.

Keywords: Conservation planning; Wildlife management, Mixed integer programming; Conservation management plans.
\end{abstract}

\section{Introduction and motivation}

We are currently experiencing a global biodoversity crisis that needs urgent attention [1]. Despite the targets set in international conventions (e.g., Aichi targets of the Convention on Biological Diversity [2]), there has been little improvement and global biodiversity continues to decline [3]. Added to the aforementioned is the fact that funding for conservation actions is typically limited, so it is essential the available financial resources are employed efficiently. Conservation planning aims to identify areas where to focus conservation efforts to ensure the long-term persistence of biodiversity. Systematic Conservation Planning (SCP, [4, chapter 14] [5]) is commonly used for designing efficient networks of Protected Areas (PAs) where to focus conservation efforts on.

To ensure the long-term persistence of biodiversity, systematic conservation planning has incorporated different elements into the design of management plans, such as spatial aspects [6, 7, 8, 9], climate change measures [10, 11], or the

\footnotetext{
${ }^{1}$ Corresponding author: Department of Statistics and Operations Research, Polytechnic University of Catalonia, Crta. de St. Lloren de Morunys a Port del Comte, km 2, Solsona, Spain (jose.salgado@upc.edu).
} 
temporal dynamics of species [12]. Spatial aspects such as connectivity not only influence the persistence of species, but also the overall ecological functioning of protected areas and the ability to manage them effectively [13]. This is usually incorporated through mathematical modification in conservation objectives [see 14, 15].

Conservation planning is usually addressed following a dichotomous approach, i.e., whether or not to select a given site, or sites, for inclusion in a protected area [16]. This approach generally results in the identification of a minimum set of areas that help achieve representation targets in an efficient way through the use of software such as Marxan [15] or Zonation [17]. However, traditional SCP methods have focused, though, on identifying priority areas but overlooked the prioritization of management actions required to ensure the long term persistence of biodiversity [16, 18, 19]. The identification of management actions within these protected areas is often disconnected from previous selection decisions, resulting in potentially inefficient solutions [20]. Multiple studies have noted the importance of prioritising alternative management actions to tackle specific threats; that provide optimised recommendations on especific actions to be implemented. These alternatives represent more efficient solutions as management actions are planned upfront and not in an $a d$ hoc fashion [see 21, 22, 23]. [20] developed a multi-action model that integrates site and action selection by performing the two steps simultaneously; thus, generating highly efficient choices of specific conservation actions that produce better results than Marxan. The authors apply a heuristic approach (specifically simulated annealing), similar to the one used in Marxan, to solve the problem. Nonetheless, heuristic approaches do not allow estimates of the quality of solutions, which is essential for measuring the efficiency of the attained management plans.

Different approaches have been used to address the prioritisation problem in systematic conservation planning. For example, greedy algorithm heuristics, mathematical optimization techniques such as linear programming (LP) and mixed integer linear programming (MIP), and greedy randomized heuristics such as simulated annealing [24]. Early algorithmic strategies used iterative methods [e.g., 25], and MIP formulations spread rapidly as a prioritisation tool [see 26, 27]. The main difficulty in using approaches based on mathematical programming is the high computational complexity of some models, which incorporate the spatial aspects of reserves with strong restrictions [see, e.g., 12, 28, 29]. Nevertheless, some of the latest studies, such as [30], have recommended avoiding the overuse of heuristics and employing exact methods wherever possible. The authors cite recent advances in mathematical programming approaches that have obtained optimal results for a wide variety of problem sizes with shorter execution times than those achieved by Marxan, which uses simulated annealing.

The fact that the prioritization approach in conservation action management has proven to be more efficient than dichotomous selection policies such as Marxan [20], together with the success of recent strategies that incorporate mathematical programming [as in 30], points up the need for a MIP model design that brings to the problem of multiple conservation action assignment the technique's advantages in terms of its flexibility of formulation and the ability to 
determine solution quality.

Here, we develop a multi-action prioritization appoach by using a MIP formulation, which incorporates connectivity considerations in the spatial prioritization of management actions. We also provide an extended version of our model by adding an expression for connectivity different management actions. In addition, we develop a procedure for the linearization of the proposed models so that they can be solved using classical integer linear programming tools. The two versions are then applied to a case study of the prioritization of conservation actions for addressing riverine threats to 45 fish species in Australia's Mitchell River catchment. To evaluate the proposed approach, we explicitly compared it with two previous heuristic aproaches: the conservation planning software Marxan and the heuristic approach developed in [20]. We expect the MIP approach to outperform heuristics in the quality of solutions and time of computing.

\section{Methods}

\subsection{The multi-action threat management planning problem (MAMP)}

In short, for a given territory and a given set of endangered species, the multi-action threat management planning problem corresponds to the problem of selecting (i) sites within this territory, known as planning units (hereafter simply "units"), and (ii) a set of actions to address the threats (against the endangered species) that occur in those units. The aim is to find an management plan that minimizes the cost of implementing the proposed actions, satisfying an ecological objective, defined as the area of each species ocurrences where they are free of threats, while at the same time, seeking for a plan to be spatially functional, by minimizing the spatial fragmentation of the selected units. Furthermore, no action may be selected without first designating the corresponding unit for monitoring to confirm that a threat is indeed present. In the remainder of this subsection we will present the mathematical notation for the formulation of the multi-action threat management planning problem using mixed integer programming.

Notation and preliminaries Let $I$ be a set of planning units such that each $i \in I$ is a basic territorial division. Also, let $s$ be the set of all species and $S_{i} \subseteq S$ the set of all species that inhabit unit $i \in I$. In addition, let $K$ be the set of all threats, $K_{i} \subseteq K$ the set of all threats that inhabit $i \in I$ and $K_{s} \subseteq K$ the set of all threats that affect species $s \in S$. We assume that there exists only one action that can abate a given threat $k \in K$, and that upon implementing this action in some unit $i \in I$, the corresponding threat $k$ in $i$ is completely abated, with $k \in K_{i}$.

To represent the economic component in our model, we define $\mathbf{c} \in \mathbb{R}_{\geq 0}^{|I| \times|K|}$ as the actions cost vector where $c_{i k}$ is the cost of applying an action to eliminate threat $k \in K_{i}$ in unit $i \in I$. Similarly, we define $\mathbf{c m} \in \mathbb{R}_{\geq 0}^{|I|}$ as a monitoring cost vector where $\mathrm{cm}_{i}$ is the monitoring cost of unit $i \in I$ in the management plan.

The spatial functionality is treated by minimizing the selected units, thus achieving a greater connection of the protection areas. To represent it, we define $\mathbf{d} \in \mathbb{R}_{\geq 0}^{|I| \times|I|}$ as the vector of pair-wise distances where $d_{i_{1}, i_{2}}$ is the distance between units $i_{1}, i_{2} \in I$, and $c v_{i_{1}, i_{2}}$ as the inverse of the square root of the distance between units $i_{1}, i_{2} \in I$ so that 
$c v_{i_{1} i_{2}}=\frac{1}{\sqrt{d_{i_{1} i_{2}}}}$. Because we will particularly work on connectivity in a freshwater conservation context, distances are measured along river corridors forcing the inclusion of closer upstream areas [see 31]. Complementary, a parameter $\beta_{1} \in[0,1]$ is defined as the penalty factor associated to the spatial fragmentation of units, similar to Boundary Length Modifier (BLM) in Marxan and as the Connectivity Strength Modifier (CSM) in [20].

As for conservation features, the local contribution, $b_{i s}$, of a given unit $i \in I$ to the benefit of the species $s \in S_{i}$ is proportional to the fraction of threats $k \in K_{s}$, that affect species $s$, for which an action is taken. Using the presented notation, we have

$$
b_{i s}= \begin{cases}\left(\frac{K_{i s}}{\left|K_{i} \cap K_{s}\right|}\right)^{\nu_{s}} & , \text { for }\left|K_{i} \cap K_{s}\right| \neq 0 \\ 1 & , \text { for }\left|K_{i} \cap K_{s}\right|=0 .\end{cases}
$$

When there are co-occurrence between the species and its threats in an unit $\left(\left|K_{i} \cap K_{s}\right|=0\right)$, we assume that all actions (against threats) are equivalent in terms of their relative contribution to local benefit. Wherever this condition is not satisfied, $b_{i s}$ is defined as 1 , the maximum possible value equivalent to carrying out all possible actions on a site for the benefit of a particular species. Whereas in any case, the domain of $b_{i s}$ ranges from $0 \leq b_{i s} \leq 1$.

As for the exponent on the right-hand side of the formula $\left(\nu_{s}\right)$, it is used for ensuring that only units where all threats affecting a given species are addressed. In this way the probability of persistence of that species would increase exponentially as we increase the number of threats being addressed. To achieve this behavior, $\nu$ must have a value greater than or equal to 1 . Very high values can produce an undesirable effect that forces all actions to be carried out so that there is a local benefit greater than 0 , however, a positive values less than 1 implies the opposite effect favoring a low total number of actions selected; while negative values would produce this same effect, but with local benefits greater than 1 . To facilitate comparison with the approach used by [20], we set this parameter as $\nu_{s}=3$.

Finally, we define $\mathbf{t} \in \mathbb{R}_{\geq 0}^{|S|}$ as the representation targets vector, where $t_{s}$ is the ecological contribution objective for each species $s \in S$.

\subsection{MIP model for the multi-action management planning problem (MAMP)}

We now formally specify MAMP, our proposed MIP model for the multi-action management planning (or "prioritization") problem. Let $\mathbf{x} \in\{0,1\}^{|I| \times|K|}$ be a vector of binary variables such that $x_{i k}=1$ if the action against threat $k \in K$ in unit $i \in I$ is selected, and $x_{i k}=0$ otherwise. Likewise, let $\mathbf{w} \in\{0,1\}^{|I|}$ be a vector of binary variables such that $w_{i}=1$ if unit $i \in I$ is taken as part of the management plan (i.e. it will be, at least, monitored), and $w_{i}=0$ otherwise. In addition, let $\mathbf{z} \in\{0,1\}^{|I| \times|S|}$ be a vector of auxiliary binary decision variables such that $z_{i s}=1$ if unit $i \in I$ contributes to the benefit of species $s \in S_{i}$ given there is an absence of threats to species $s$ on unit $i\left(\left|K_{i} \cap K_{s}\right|=0\right)$, otherwise $z_{i s}=0$. The purpose of this variable is to quantify the contributions to the local benefit of a species that occur simply by selecting a unit as part of the management plan (monitoring). 
Having defined the variables, we can now rewrite the local benefit function set above. In a unit $i \in I$ for a species $s \in S_{i}$, it is given by

$$
b_{i s}= \begin{cases}\left(\frac{\sum_{k \in K_{i} \cap K_{s}} x_{i k}}{\left|K_{i} \cap K_{s}\right|}\right)^{3} & , \text { for }\left|K_{i} \cap K_{s}\right| \neq 0 \\ z_{i s} & , \text { for }\left|K_{i} \cap K_{s}\right|=0,\end{cases}
$$

and therefore, the total benefit for a species $s$ can be written as

$$
b_{s}=\sum_{i \in I:\left|K_{i} \cap K_{s}\right| \neq 0}\left(\frac{\sum_{k \in K_{i} \cap K_{s}} x_{i k}}{\left|K_{i} \cap K_{s}\right|}\right)^{3}+\sum_{i^{\prime} \in I:\left|K_{i} \cap K_{s}\right|=0} z_{i^{\prime} s} .
$$

Given that a solution is characterized by the vector of variables mentioned above, a particular solution of the model is encoded by a triplet $(\mathbf{w}, \mathbf{x}, \mathbf{z})$. In this way, the total cost function of such a solution $(\mathbf{w}, \mathbf{x}, \mathbf{z})$, is given by

$$
C(\mathbf{w}, \mathbf{x}, \mathbf{z})=\sum_{i \in I} \sum_{t \in K_{i}} c_{i k} x_{i k}+\sum_{i \in I} c m_{i} w_{i}
$$

i.e. the sum of the action costs plus the monitoring costs the management plan. Note that in order to perform an action on unit i, it must first be selected for monitoring. Similarly, the fragmentation function of units of such a solution ( $\mathbf{w}, \mathbf{x}, \mathbf{z})$, is given by

$$
F(\mathbf{w}, \mathbf{x}, \mathbf{z})=\sum_{i_{1} \in I} \sum_{i_{2} \in I: i_{1} \neq i_{2}} w_{i_{1}}\left(1-w_{i_{2}}\right) c v_{i_{1} i_{2}}
$$

This expression is commonly found in mathematical conservation models to represent the spatial aspects [6], does not impose total connectivity of all of the units but rather allows a tradeoff to be sought between the cost of implementing the management plan and the degree of its fragmentation, as a way of improving the decision-making process. More specifically, it penalizes the non-incorporation of units with non-zero connectivity penalties, that is, units that are relatively close to each other, also allowing the incorporation of asymmetric relations between units [32].

Given all of the above, the model MAMP can be formulated as the following optimization problem;

$$
\begin{array}{rlrl}
\operatorname{MAMP}^{*}\left(\mathbf{w}^{*}, \mathbf{x}^{*}, \mathbf{z}^{*}\right)= & \min & & \\
\text { s.t. } & & \sum_{i \in I:\left|K_{i} \cap K_{s}\right| \neq 0}\left(\frac{\sum_{k \in K_{i} \cap K_{s}} x_{i k}}{\left|K_{i} \cap K_{s}\right|}\right)^{3}+\sum_{i \in I:\left|K_{i} \cap K_{s}\right|=0} z_{i s} \geq t_{s}, & \\
& \sum_{k \in K_{i}} x_{i k} \leq\left|K_{i}\right| w_{i}, & & \\
& \sum_{s \in S_{i}} z_{i s} \leq\left|S_{i}\right| w_{i}, & \forall i \in I \\
& & \forall i \in I \\
\mathbf{w} \in\{0,1\}^{|I|}, \mathbf{x} \in\{0,1\}^{|I| \times|K|}, \mathbf{z} \in\{0,1\}^{|I| \times|S|} . &
\end{array}
$$

The two concepts described above, the total cost and the fragmentation of units, correspond to the objective function and are encoded in (MAMP.1, where $\beta_{1}$ is the penalty factor of fragmentation of units. In cases where connectivity is not 
relevant, this factor is assumed to be 0 . As regards the restrictions, constraint (MAMP.2) ensures the representation target $t_{s}$ for each species is satisfied. This target can be achieved directly by selecting units where the species is free of threats, that is, where there are no co-occurrences between this species and its threats (i.e., $\left(\left|K_{i} \cap K_{s}\right|=0\right.$ ), or by selecting units and actions that produce an equivalent sum of local contributions. The non-linear nature of the benefit function $\left(b_{i} s\right)$ is discussed later in this section. Constraint (MAMP.3 imposes that for an action to be implemented in unit $i \in I$, that unit must be part of the management plan $\left(w_{i}=1\right)$. In similar fashion, constraint (MAMP.4) requires that for a unit $i \in I$ to contribute to the objective for species $s \in S_{i}$, that unit must be part of the management plan $\left(w_{i}=1\right)$. Finally, constraint (MAMP.5) defines the nature of the variables.

Note that the multi-action assignment problem is structurally much more complex than the commonly used planning problem involving a single action [21]. In our model, the solution space in the worst-case scenario $\left(K_{i}=K, \forall i \in I\right)$ is incremented from $2^{I}$ to $(K+1)^{I}$, where $K$ is the number of threats and $I$ the number of planning units. We therefore assume that the model should be limited to addressing a large number of threats in order to avoid the exponential growth of the problem and the consequent difficulties in finding a good solution [24].

Due to the model developed in [20] and the Marxan software both use heuristic solution approaches (simulated annealing), the non-linearity of some of their components poses no problem for the solution process, while in the case of linear programming, the application of linearization methods is necessary. Specifically in MAMP, both $F(\mathbf{w}, \mathbf{x}, \mathbf{z})$ and $b_{i s}$ are non-linear functions with respect to variables, but with a different nature. On the one hand, $F(\mathbf{w}, \mathbf{x}, \mathbf{z})$ is a quadratic function given by a multiplication of binary variables, this non-linearity can be addressed by induction of the so-called boolean quadratic polytope, used recently in the context of conservation planning by [30]. A detailed description of this methodology applied to our model is presented in the Appendix B. Moreover, $b_{i s}$ is nonlinear given by its cubic exponent, which is addressed by means of a piece-wise function, that incorporates the creation of a new set of variables and restrictions as well as a new parameter $\gamma$ that indicates the number of linear segments that the piece-wise function has. In this sense, we use the strategy coded in the IloPiecewiseLinear function of IBM ILOG CPLEX 12.6.3 while the explanation of this linearization methodology is described in the Appendix B.

\subsubsection{Extended MAMP model (MAMP-E)}

To further address connectivity issues in our MAMP model, we extended it to also account for connectivity within management actions. In this way, we define the fragmentation of units, we incorporate the concept called fragmentation of action $k, F A_{k}(\mathbf{w}, \mathbf{x}, \mathbf{z})$, which calculates the fragmentation within the same management action, it is given by

$$
F A_{k}(\mathbf{w}, \mathbf{x}, \mathbf{z})=\sum_{i_{1} \in I} \sum_{i_{2} \in I: i_{1} \neq i_{2}} x_{i_{1} k}\left(1-x_{i_{2} k}\right) c v_{i_{1} i_{2}} .
$$

The introduction of this function seeks to improve management plans by grouping the units were a given action has been selected, so management actions are spatially cumpled, achieving benefits through economies of scale and greater 
efficiency in actions, reducing for example the likelihood of re-infection. This function can be generalized for all actions $(F A(\mathbf{w}, \mathbf{x}, \mathbf{z}))$ in the form,

$$
F A(\mathbf{w}, \mathbf{x}, \mathbf{z})=\sum_{k \in K} F A_{k}(\mathbf{w}, \mathbf{x}, \mathbf{z}) .
$$

The incorporation of this function in our MAMP model generates a new extended model, denoted as MAMP-E, which can be declared as follows:

$$
\operatorname{MAMP}-E^{*}\left(\mathbf{w}^{*}, \mathbf{x}^{*}, \mathbf{z}^{*}\right)=\min \quad C(\mathbf{w}, \mathbf{x}, \mathbf{z})+\beta_{1} F(\mathbf{w}, \mathbf{x}, \mathbf{z})+\beta_{2} F A(\mathbf{w}, \mathbf{x}, \mathbf{z})
$$

$$
\text { s.t. MAMP.2 - MAMP.5, }
$$

where $\beta_{2} \in[0,1]$ is defined as the penalty factor associated to the spatial fragmentation of actions, which has the same goal than $\beta_{1}$ in (MAMP.1). We assume equal importance in the connectivity of all actions. The idea behind this extended formulation is to determine the impact on the solutions of the extended model's requirement to improve connectivity not only between units but also between the actions taken within them. Both models allow us to analyze the effect of connectivity requirements on the costs as well as on the ecological benefits to the species.

\subsection{Solution comparison measures}

To facilitate comparisons of the management plans produced by the different parameters, a series of measures are defined and calculated for each solution $(\mathbf{w}, \mathbf{x}, \mathbf{z})$ generated. These measures are listed below.

Cost efficency $E(\mathbf{w}, \mathbf{x}, \mathbf{z})$ : Defined as 1 minus the ratio of the total cost function of the plan $C(\mathbf{w}, \mathbf{x}, \mathbf{z})$ with respect to the maximum possible cost $C_{T}$; note that $C_{T}$ is a fixed value and it corresponds to the cost that would be incurred if all actions were implemented and all units were selected for incorporation into the management plan, and is given by $\sum_{i \in I} \sum_{k \in K_{i}} c_{i k}+\sum_{i \in I} c m_{i}$ :

$$
E(\mathbf{w}, \mathbf{x}, \mathbf{z})=1-\frac{C(\mathbf{w}, \mathbf{x}, \mathbf{z})}{C_{T}}=1-\frac{\sum_{i \in I} \sum_{k \in K_{i}} c_{i k} x_{i k}+\sum_{i \in I} c m_{i} w_{i}}{\sum_{i \in I} \sum_{k \in K_{i}} c_{i k}+\sum_{i \in I} c m_{i}} .
$$

Degree of connectivity of units $D C U(\mathbf{w}, \mathbf{x}, \mathbf{z}): \quad$ Defined as 1 minus the ratio of $F(\mathbf{w}, \mathbf{x}, \mathbf{z})\left(\beta_{1}\right)$, with respect to $F_{\max }$; where $F(\mathbf{w}, \mathbf{x}, \mathbf{z})\left(\beta_{1}\right)$ corresponds to the fragmentation of units given by the penalty factor $\beta_{1}$, and $F_{\max }$ corresponds to the maximum fragmentation of units found, across all values of $\beta_{1}$, using either MAMP, MAMP-E, the approach proposed in [20], or Marxan:

$$
D C U(\mathbf{w}, \mathbf{x}, \mathbf{z})=1-\frac{F(\mathbf{w}, \mathbf{x}, \mathbf{z})\left(\beta_{1}\right)}{F_{\max }}=1-\frac{\sum_{i_{1} \in I} \sum_{i_{2} \in I: i_{1} \neq i_{2}} c v_{i_{1}, i_{2}} w_{i_{1}}\left(1-w_{i_{2}}\right)}{F_{\max }} .
$$

Degree of connectivity of action $k D C A_{k}(\mathbf{w}, \mathbf{x}, \mathbf{z})$ : Defined as 1 minus the ratio of $F A_{k}(\mathbf{w}, \mathbf{x}, \mathbf{z})\left(\beta_{1}, \beta_{2}\right)$ with respect to $\left(F A_{k-\max }\right)$; where $F A_{k}(\mathbf{w}, \mathbf{x}, \mathbf{z})\left(\beta_{1}, \beta_{2}\right)$ corresponds to the fragmentation of action $k$ for a given penalty factors of 
$\beta_{1}$ and $\beta_{2}$, and $F A_{k-\max }$ corresponds to the maximum fragmentation of action $k$, across all values of $\beta_{1}$ and $\beta_{2}$, using either MAMP, MAMP-E, the approach proposed in [20], or Marxan:

$$
D C A_{k}(\mathbf{w}, \mathbf{x}, \mathbf{z})=1-\frac{F A_{k}(\mathbf{w}, \mathbf{x}, \mathbf{z})\left(\beta_{1}, \beta_{2}\right)}{F A_{k-\max }}=1-\frac{\sum_{i_{1} \in I} \sum_{i_{2} \in I: i_{1} \neq i_{2}} c v_{i_{1}, i_{2}} x_{i_{1}, k}\left(1-x_{i_{2}, k}\right)}{F A_{k-\max }} .
$$

Average degree of connectivity of actions $\overline{D C A}(\mathbf{w}, \mathbf{x}, \mathbf{z})$ : Defined as the average of the values of $D C A_{k}(\mathbf{w}, \mathbf{x}, \mathbf{z})$ with respect to the set of actions $K$ :

$$
\overline{D C A}(\mathbf{w}, \mathbf{x}, \mathbf{z})=\frac{\sum_{k \in K} D C A_{k}(\mathbf{w}, \mathbf{x}, \mathbf{z})}{|K|} .
$$

Average benefit $\bar{B}(w, x, z)$ : Defined as the average value of the benefit achieved across all species encoded by $S$ :

$$
\bar{B}(\mathbf{w}, \mathbf{x}, \mathbf{z})=\frac{\sum_{s \in S}\left(\frac{b_{s}}{\left|I_{s}\right|}\right)}{|S|}
$$

As can be seen, the degrees of connectivity are defined to be inversely proportional to the corresponding fragmentation penalty factor $\left(\beta_{1}\right.$ and/or $\left.\beta_{2}\right)$, in the same way, an inverse relationship also characterizes the $C(\mathbf{w}, \mathbf{x}, \mathbf{z})$ with respect to $E(\mathbf{w}, \mathbf{x}, \mathbf{z})$.

\subsection{Case study}

We used the Mitchell River catchment in Northern Australia as a case study to compare the model proposed here against two alternative approaches to multi-action planning already existing for the catchment [see[20]. The area that spans over 71,630 $\mathrm{km}^{2}$, was divided into 2316 subcatchments or units planning. The study focussed on 45 freshwater fish species whose distribution within the catchment is depicted in 1(a), Four major threats to these species were considered: the water buffalo (Bubalis bubalis), the cane toad (Rhinella marina), river flow alteration (caused by impoundments, channels for water extractions and levee banks) and grazing land use [see 20, for further details]. The spatial distribution of these threats is also shown in the figure 1(b) The two subfigures further indicate that each sub-catchment has different species and threat densities, demconstrating the need for an optimization model to design a multi-action management plan that is both functional and economically efficient. Full information on the relationships between these species and threats and the presence of each one in the Mitchell River catchment may be freely accessed at https://doi.org/10.6084/ m9.figshare.1396588.v2 and https://doi.org/10.6084/m9.figshare.1396587.v2, respectively.

\subsection{Problem parameter values}

Based on the parameters for the multi-action managment planning problem used by [20], a number of parameters are determined as detailed in Table 1 The cost parameters are set of $1[k U S]$. This value is set to rule out the cost effect on the connectivity of the resulting management plan. The representation target $\left(t_{s}\right)$ is set at 100 spatial units for each species. 


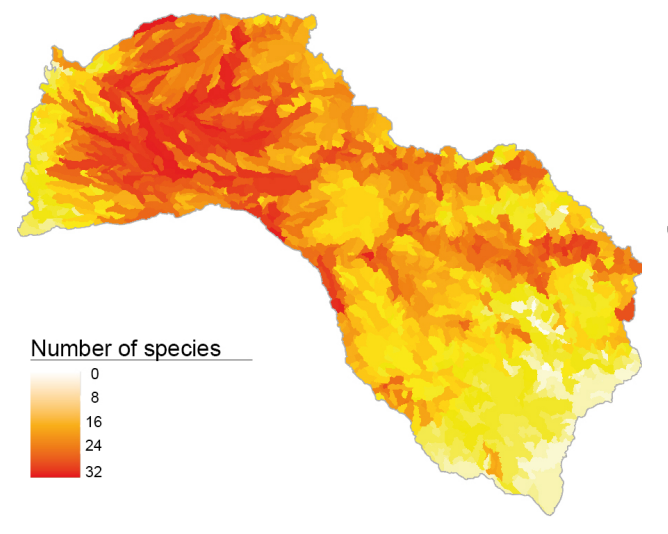

(a) Species distribution

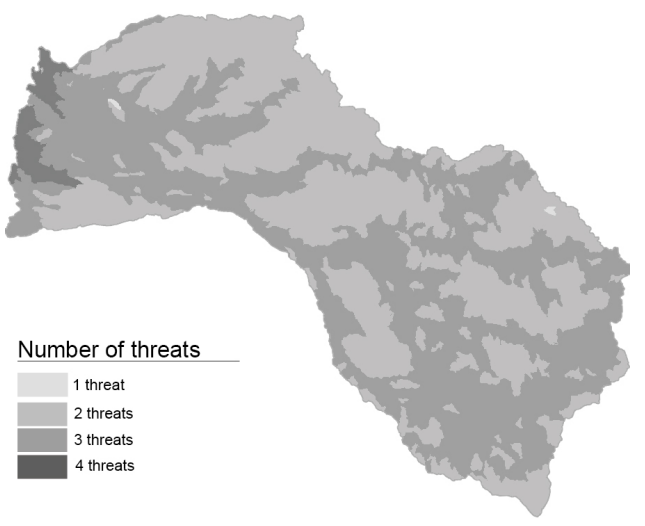

(b) Threat distribution

Figure 1: Spatial distribution of species richness (a) and species and threats (b) in the Mitchell River catchment, northern Australia. Darker colours show hier richness and number of threats respectively.

This guarantees the selection of at least 100 planning units where there is a total contribution equivalent to the choice of 100 units free of threats to that species. For those species inhabiting fewer subcatchments, it is deemed necessary to select all of the units where the species are found, thereby eliminating all real threats to them.

\begin{tabular}{lc}
\hline Parameters & Value \\
\hline Monitoring Cost $\left(c m_{i}, i \in I\right)$ & 1 \\
Threat elimination cost $\left(c_{i k}, i \in I, k \in K_{i}\right)$ & 1 \\
Representation targets by species $\left(t_{s}, s \in S\right)$ & 100 \\
Linearisation breakpoints $\left(b p_{m}, m \in \mathbb{N}_{\leq \gamma+1}\right)$ & $\in\{0,0.33,0.66,1\}$ \\
Penalty factor of fragmentation of units $\left(\beta_{1}\right)$ & $\in\{0,0.2, \ldots, 1,1.5,2, \ldots, 12\}$ \\
Penalty factor of fragmentation of actions $\left(\beta_{2}\right)$ & $\in\{0,0.2, \ldots, 1,1.5,2, \ldots, 12\}$ \\
\hline
\end{tabular}

Table 1: Parameters used in solving the MAMP and MAMP-E models.

The reader shall be aware that the non-linearity of $b_{i s}$ was addressed by means of a piece-wise linear function scheme. In such strategy, the higher the number of linear segments $(\gamma)$, the stricter the approximation of the corresponding cubic function. Nonetheless, high values $\gamma$ significantly increase the resulting computational difficulty, yielding higher computational times. Preliminary tests performed for different values of $\gamma$ showed that $\gamma=3$ offered a suitable balance between computational tractability and reasonable running times; so we set $\gamma=3$ across all analyses (Appendix C). Therewith, we can define $m \in \mathbb{N}_{\leq \gamma+1}$ as the set of breakpoints of the piece-wise function (note that the number of breakpoints is one more than the number of linear segments), so if we consider breakpoints equidistant for $\gamma=3$ and that the domain of the cubic function is $[0,1]$, it is equivalent to specifying the set of breakpoints as $b p_{m}=\{0,0.33,0.66,1\}$.

Regarding the spatial functionality objective (connectivity), a range of different penalty factors $\beta_{1} \in\{0,0.2, \ldots, 1,1.5$, $2, \ldots, 12\}$ were tested. The limit is set to 12 because an increase in the beta factor, from that value, does not add an im- 


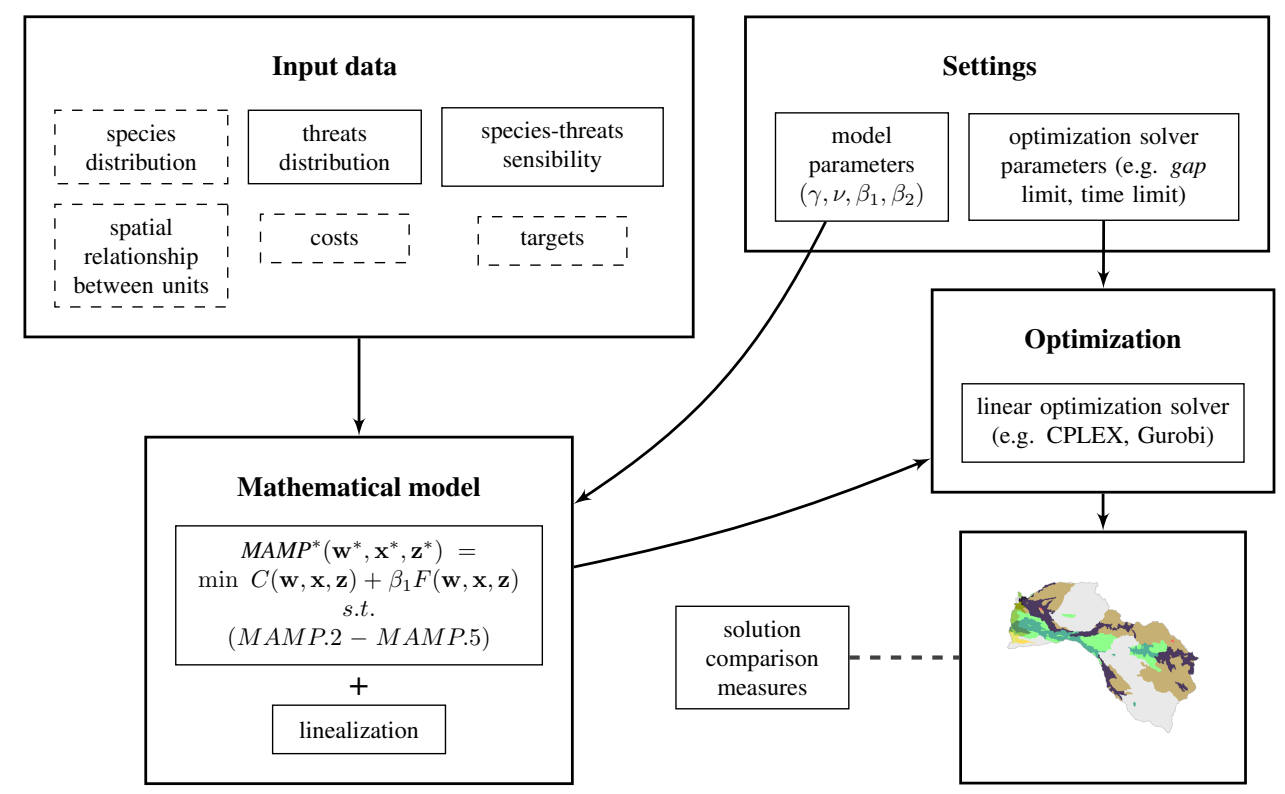

Figure 2: Conceptual scheme of the proposed framework

provement in the degree of connectivity of the units $(D C U)$. In the same way, the MAMP-E model is solved for a broad range of penalty factors $\beta_{2} \in\{0,0.2, \ldots, 1,1.5,2, \ldots, 12\}$ and using a fixed penalty factor of fragmentation of units, $\beta_{1}=0.8$. This $\beta_{1}$ factor was selected as the best trade-off between cost and connectivity in the MAMP model. This exploration of beta values is carried out to evaluate the relationship between the cost and connectivity, similarly as done when calibrating the BLM in Marxan [see 33].

Experimental settings The two proposed MIP models were written in the $\mathrm{C}++$ programming language (code available in Appendix D) and solved using the CPLEX 12.6.3 solver with Concert Technology running on an Intel Core i7-5820k $3.3 \mathrm{GHz}$ 12-core processor with $32 \mathrm{~GB}$ of DDR4 RAM. The solver was parameterized to stop once the execution time reached 6 hours (or earlier if an optimal solution was found). To improve the solver performance, CPLEX polishing strategy was applied once a 20,000-second mark was passed. On the other hand, Marxan was run with its default values (10 replicates and $10^{6}$ iterations). The quality of the solutions was measured using the gap criterion, defined as the percentage difference between the relaxed (lower bound) and best (upper bound) solutions found by CPLEX; hence, the the lower the value of the gap, the closer the solution of the optimal one.

A conceptual diagram of our multi-action prioritisation approach is displayed in Figure 2. As can be seen from the figure, the first block corresponds to the input data, whose blocks with dotted lines inside indicate the data that is commonly used as input for the Marxan software. With respect to the second block (settings), it is composed of parameters specific to the model $\left(\beta_{1}, \beta_{2}, \gamma, \nu\right)$, whose selection or calibration of values will be explained in the following section, and, the parameters of the optimization solver, which include the strategies followed for the search for better solutions 


\begin{tabular}{cccccccccc}
\hline$\beta_{1}$ & gap (\%) & $E$ & $D C U$ & $D C A_{1}$ & $D C A_{2}$ & $D C A_{3}$ & $D C A_{4}$ & $\overline{D C A}$ & $\bar{B}$ \\
\hline 0 & 0.1 & 0.87 & 0 & 0.47 & 0.36 & 0.51 & 0.23 & 0.39 & 0.4 \\
0.2 & 0.19 & 0.87 & 0.21 & 0.47 & 0.53 & 0.56 & 0.39 & 0.49 & 0.41 \\
0.4 & 0.23 & 0.86 & 0.32 & 0.47 & 0.6 & 0.56 & 0.44 & 0.52 & 0.41 \\
0.6 & 0.52 & 0.85 & 0.52 & 0.47 & 0.59 & 0.6 & 0.45 & 0.53 & 0.41 \\
0.8 & 0.67 & 0.85 & 0.57 & 0.47 & 0.58 & 0.57 & 0.43 & 0.51 & 0.41 \\
1 & 0.87 & 0.85 & 0.58 & 0.47 & 0.58 & 0.56 & 0.43 & 0.51 & 0.41 \\
1.5 & 0.89 & 0.84 & 0.66 & 0.47 & 0.56 & 0.59 & 0.41 & 0.51 & 0.41 \\
2 & 1.07 & 0.83 & 0.71 & 0.47 & 0.55 & 0.57 & 0.39 & 0.49 & 0.41 \\
2.5 & 1.03 & 0.81 & 0.78 & 0.47 & 0.46 & 0.54 & 0.36 & 0.46 & 0.42 \\
3 & 0.95 & 0.8 & 0.79 & 0.47 & 0.46 & 0.55 & 0.35 & 0.46 & 0.42 \\
3.5 & 0.98 & 0.79 & 0.84 & 0.47 & 0.44 & 0.53 & 0.34 & 0.44 & 0.42 \\
4 & 1.09 & 0.77 & 0.88 & 0.47 & 0.42 & 0.55 & 0.3 & 0.43 & 0.41 \\
4.5 & 0.87 & 0.76 & 0.9 & 0.47 & 0.4 & 0.52 & 0.3 & 0.42 & 0.42 \\
5 & 0.91 & 0.75 & 0.91 & 0.47 & 0.41 & 0.52 & 0.29 & 0.42 & 0.42 \\
5.5 & 0.84 & 0.74 & 0.92 & 0.47 & 0.36 & 0.5 & 0.25 & 0.39 & 0.42 \\
6 & 0.81 & 0.74 & 0.92 & 0.47 & 0.37 & 0.51 & 0.26 & 0.4 & 0.42 \\
6.5 & 0.78 & 0.74 & 0.93 & 0.47 & 0.35 & 0.51 & 0.27 & 0.4 & 0.41 \\
7 & 0.78 & 0.74 & 0.93 & 0.47 & 0.37 & 0.53 & 0.27 & 0.41 & 0.41 \\
7.5 & 0.75 & 0.74 & 0.94 & 0.47 & 0.35 & 0.51 & 0.26 & 0.4 & 0.41 \\
8 & 0.83 & 0.7 & 0.97 & 0.47 & 0.36 & 0.51 & 0.26 & 0.4 & 0.41 \\
8.5 & 0.86 & 0.7 & 0.97 & 0.47 & 0.35 & 0.52 & 0.26 & 0.4 & 0.41 \\
9 & 0.85 & 0.7 & 0.98 & 0.47 & 0.37 & 0.53 & 0.25 & 0.4 & 0.41 \\
9.5 & 0.81 & 0.7 & 0.98 & 0.47 & 0.36 & 0.52 & 0.24 & 0.4 & 0.41 \\
10 & 0.81 & 0.7 & 0.98 & 0.47 & 0.37 & 0.53 & 0.25 & 0.4 & 0.41 \\
10.5 & 0.81 & 0.7 & 0.98 & 0.47 & 0.36 & 0.52 & 0.26 & 0.4 & 0.41 \\
11 & 0.77 & 0.7 & 0.98 & 0.47 & 0.37 & 0.53 & 0.26 & 0.4 & 0.41 \\
11.5 & 0.8 & 0.7 & 0.98 & 0.47 & 0.36 & 0.5 & 0.25 & 0.4 & 0.41 \\
12 & 0.79 & 0.7 & 0.98 & 0.47 & 0.36 & 0.53 & 0.25 & 0.4 & 0.41 \\
\hline $1-$ & & & &
\end{tabular}

$1=$ Water buffalo, $2=$ Cane toad, $3=$ River flow, $4=$ Grazing

Table 2: Results of MAMP model using exact solution methods. The rows from top to bottom give the specific values generated for successively higher spatial functionality requirements imposed by the complete range of penalty factors $\left(\beta_{1}\right)$ so that the different solutions can be readily compared

(e.g. polishing strategy). The third block includes the mathematical model (both MAMP and its extension), adding the transformations necessary to obtain a linear model. The fourth block assumes the resolution of the model by means of an optimization solver incorporating the adjustments previously established, ending with the obtaining and comparison of results.

\section{Computational results for MAMP and MAMP-E}

Our experiments are aimed essentially at evaluating three fundamental aspects: (1) the tradeoff between the management plan cost-benefit index and the degree of connectivity of units $(D C U)$, the latter a measure of the plan's spatial functionality, which is then compared to the results obtained by [20] and Marxan; (2) the computational efficiency of the MAMP and MAMP-E models and (3) an understanding of the behaviour of the average benefit $(\bar{B})$ generated by the two formulations as the penalty factors $\beta_{1}$ and $\beta_{2}$ are increased. 
The results obtained for the different parameters by the exact solution method with the MAMP model are reported in Table 2, wherein is observed that regardless of the imposed spatial functionality level, the solutions are of high quality with an average gap of less than $1 \%$. The cost efficency $(E)$ clearly declines as $\beta_{1}$ increases, reflecting an increase in the number of actions and monitoring and treated units in order to improve connectivity. Despite this increase in the degree of connectivity of units $(D C U)$, average degree of connectivity of actions $(\overline{D C A})$ and average benefit $(\bar{B})$ both remain relatively constant for any given $\beta_{1}$ value, which is explained by the constraint (MAMP.2), that explicitly establishes the need to achieve the representation targets for all species $\left(t_{s}\right)$.

On the other hand, the results obtained with MAMP-E are displayed in Table 3 . As can be seen, compared to MAMP the average gap is greater, reaching a maximum of 3.77\%. Regarding the average degree of connectivity of the actions $(\overline{D C A})$, its values are higher than for MAMP due to this variable's greater importance in the extended model's objective function. This higher connectivity was achieved at the expenses of larger number of planning units under direct intervention. Also, the behavior of this criterion when increasing the penalty factor associated to actions $\left(\beta_{2}\right)$ is different for each threat, due particularly to the threats' respective spatial distributions. For example, the spatial distribution of water buffaloes is small and centred in the lower region of the Mitcheell Rivercatchment, while grazing is distributed throughout the basin. The foregoing, because there is a more significant benefit from minimizing the fragmentation of this latter threat, and therefore, its $D C A$ increases with increasing $\beta_{2}$. While on the other hand, unlike MAMP, the average benefit achieved for each species $(\bar{B})$ increases with $\beta_{2}$, because more actions are being taken to improve within-actions connectivity.

Regarding to the computation aspect, six hours of computation time were enough for the MAMP model to achieve an optimality gap of less than $1 \%$ at any connectivity functionality level. In comparison with [20], whose heuristic needed 16 hours to attain a gap of $3 \%$ (with regards to the bounds provided of the MAMP model), our MIP approach attain an equivalent solution in an average of fewer than 1,000 seconds; this is a significant improvement in computational efficiency. The extended version, MAMP-E, managed to reach a 3\% gap in 1,700 seconds; which is reasonable considering the larger size of the problem that had to be solved.

The comparison of efficiency across different methods showed that, for any degree of connectivity of units $(D C U)$, the MAMP model outperforms both [20] and Marxan, generating better conservation action plans from the standpoint of cost as well as functionality (Figure 3(a). As for MAMP-E, since it cannot be compared with the two approximate methods, we only contrast it with MAMP. In this way, we observe a relative decrease in the cost efficency $(E)$ for the extended model as a growing number of spatially connected actions increases the cost relative to unit connectivity (Figure 3(b) $)$. This tendency stems from the fact that the main objective of MAMP-E is the connectivity of actions.

The spatial distribution of the actions selected by the MAMP model when increasing the penalty factor associated to 


\begin{tabular}{cccccccccc}
\hline$\beta_{2}$ & gap (\%) & $E$ & $D C U$ & $D C A_{1}$ & $D C A_{2}$ & $D C A_{3}$ & $D C A_{4}$ & $\overline{D C A}$ & $\bar{B}$ \\
\hline 0 & 0.66 & 0.85 & 0.57 & 0.47 & 0.58 & 0.57 & 0.43 & 0.51 & 0.41 \\
0.2 & 0.75 & 0.85 & 0.56 & 0.47 & 0.63 & 0.67 & 0.54 & 0.58 & 0.42 \\
0.4 & 1.26 & 0.84 & 0.56 & 0.51 & 0.68 & 0.67 & 0.59 & 0.61 & 0.43 \\
0.6 & 0.99 & 0.83 & 0.56 & 0.51 & 0.76 & 0.68 & 0.64 & 0.65 & 0.44 \\
0.8 & 1.16 & 0.83 & 0.57 & 0.53 & 0.78 & 0.71 & 0.67 & 0.67 & 0.45 \\
1 & 1.25 & 0.81 & 0.6 & 0.4 & 0.81 & 0.7 & 0.69 & 0.65 & 0.45 \\
1.5 & 1.44 & 0.8 & 0.64 & 0.4 & 0.81 & 0.71 & 0.72 & 0.66 & 0.47 \\
2 & 2.66 & 0.78 & 0.67 & 0.25 & 0.83 & 0.68 & 0.74 & 0.62 & 0.48 \\
2.5 & 2.79 & 0.77 & 0.7 & 0.25 & 0.83 & 0.68 & 0.76 & 0.63 & 0.49 \\
3 & 3.71 & 0.76 & 0.7 & 0.25 & 0.84 & 0.69 & 0.77 & 0.64 & 0.5 \\
3.5 & 2.98 & 0.71 & 0.78 & 0.25 & 0.87 & 0.64 & 0.83 & 0.65 & 0.54 \\
4 & 3.82 & 0.69 & 0.78 & 0.2 & 0.88 & 0.66 & 0.83 & 0.64 & 0.55 \\
4.5 & 2.12 & 0.67 & 0.81 & 0.2 & 0.9 & 0.64 & 0.85 & 0.64 & 0.55 \\
5 & 2.59 & 0.64 & 0.83 & 0.17 & 0.91 & 0.63 & 0.86 & 0.64 & 0.57 \\
5.5 & 2.58 & 0.6 & 0.86 & 0.17 & 0.93 & 0.64 & 0.89 & 0.66 & 0.59 \\
6 & 3.38 & 0.59 & 0.88 & 0.17 & 0.92 & 0.63 & 0.91 & 0.66 & 0.6 \\
6.5 & 2.57 & 0.52 & 0.92 & 0.17 & 0.96 & 0.56 & 0.93 & 0.65 & 0.65 \\
7 & 2.16 & 0.52 & 0.92 & 0.17 & 0.96 & 0.56 & 0.93 & 0.65 & 0.65 \\
7.5 & 1.97 & 0.52 & 0.92 & 0.17 & 0.96 & 0.56 & 0.93 & 0.66 & 0.65 \\
8 & 2.52 & 0.51 & 0.92 & 0.17 & 0.96 & 0.56 & 0.94 & 0.66 & 0.66 \\
8.5 & 1.84 & 0.51 & 0.92 & 0.17 & 0.96 & 0.56 & 0.93 & 0.66 & 0.66 \\
9 & 1.92 & 0.51 & 0.92 & 0.17 & 0.96 & 0.56 & 0.94 & 0.66 & 0.66 \\
9.5 & 2.34 & 0.5 & 0.93 & 0.17 & 0.96 & 0.56 & 0.94 & 0.66 & 0.67 \\
10 & 2.68 & 0.49 & 0.93 & 0.04 & 0.96 & 0.54 & 0.94 & 0.62 & 0.68 \\
10.5 & 2.86 & 0.44 & 0.94 & 0.04 & 0.96 & 0.43 & 0.94 & 0.59 & 0.69 \\
11 & 3.33 & 0.45 & 0.94 & 0.04 & 0.96 & 0.5 & 0.94 & 0.61 & 0.7 \\
11.5 & 3.77 & 0.47 & 0.94 & 0.04 & 0.96 & 0.52 & 0.94 & 0.61 & 0.69 \\
12 & 3.13 & 0.44 & 0.95 & 0.04 & 0.96 & 0.43 & 0.95 & 0.59 & 0.7 \\
\hline $1=$ & & & &
\end{tabular}

$1=$ Water buffalo, $2=$ Cane toad, $3=$ River flow, $4=$ Grazing

Table 3: Results of MAMP-E model using exact solution methods. The rows from top to bottom give the specific values generated for successively higher spatial functionality requirements imposed by the complete range of penalty factors $\left(\beta_{2}\right)$ with the penalty factor associated to the spatial fragmentation of units held constant at $\left(\beta_{1}=0.8\right)$ 


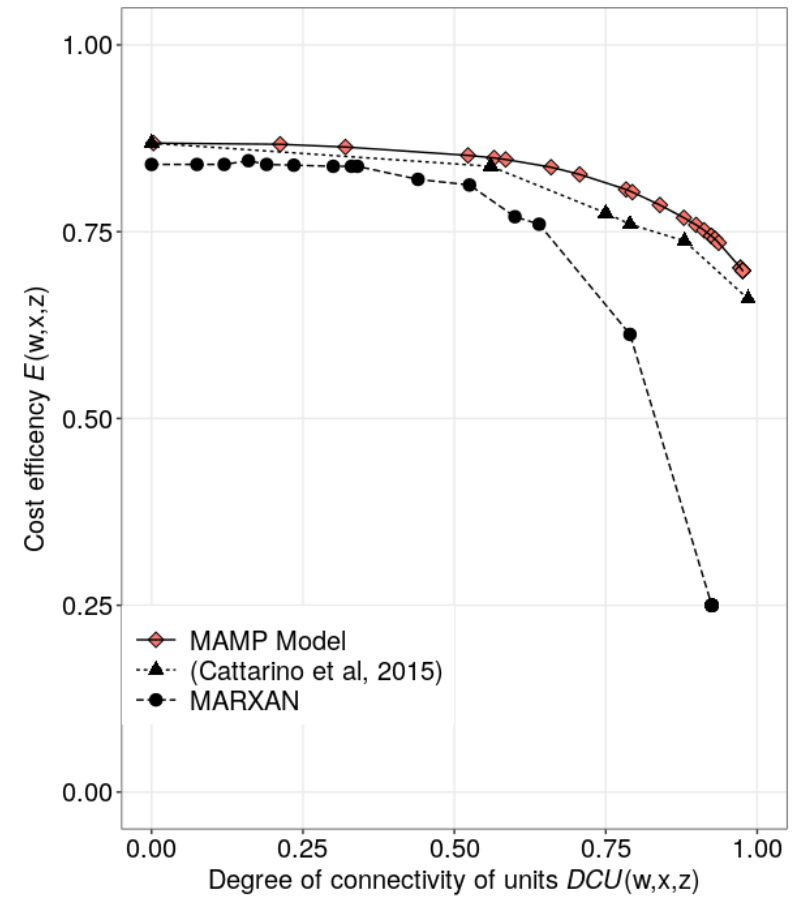

(a)

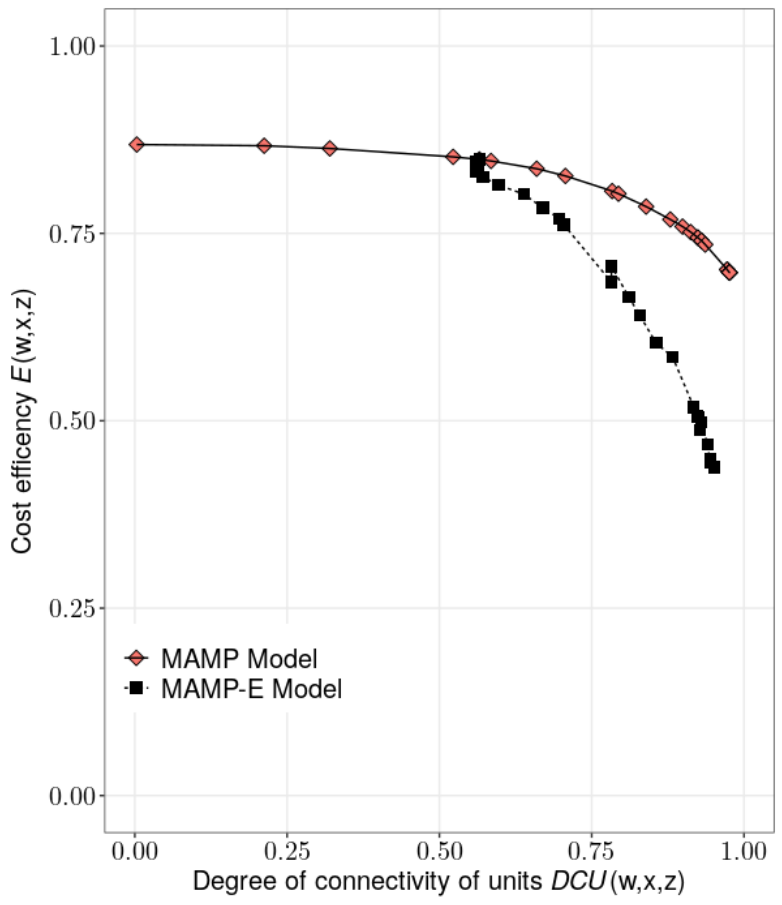

(b)

Figure 3: Behaviour of the cost efficency $(E)$ with respect to changes in degree of connectivity of units $(D C U)$ for different solution methods used in multi-action conservation prioritization models. The graph in panel (a) compares the proposed MAMP model with [20] and Marxan. The graph in panel (b) compares the MAMP model with its extension, MAMP-E.

the spatial fragmentation of units $\left(\beta_{1}\right)$ shows a greater selection of units (thus increasing connectivity), where much of these new units are selected for monitoring (Figure 4). This implies that the actions towards the most populated threats have more and more freedom to move in order to achieve this new spatial requirement, even assuming a higher cost of the management plan. The movement of actions under the increased requirement of connectivity is limited by the actions that must be invariably carried out to achieve the target representation of scarce species. On the other hand, for MAMP-E model, the spatial distribution of the selected actions by increasing the penalty factor associated to the spatial fragmentation of actions $\left(\beta_{2}\right)$, shows the selection of actions in units that have a greater number of threats, benefiting from their spatial distribution.

When comparing the number of actions selected for each spatial unit, Marxan always showed higher numbers and, therefore, higher total cost per unit than the other tested methods (see Figure 5). Moreover, for three threats (cane toad, alterations in river flow, grazing), the MAMP model is more efficient, than the other two tested methods, in the use of actions when $\beta_{1}>0$, and equally efficient when $\beta_{1}=0$ (because it was in all cases the same solution). In the case of the water buffalo, the MAMP model produces solutions that are not better than in [20]. Allowing a greater balance between the other actions (achieving more efficiency in them). Moreover, being the rarest threat, the difference between 


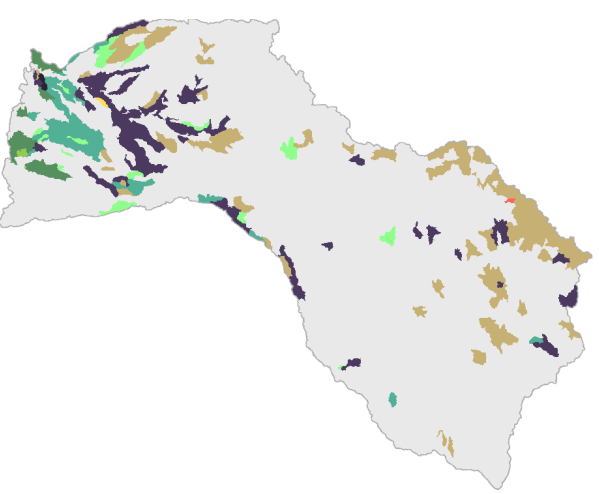

(a) $\beta_{1}=0, \beta_{2}=0$

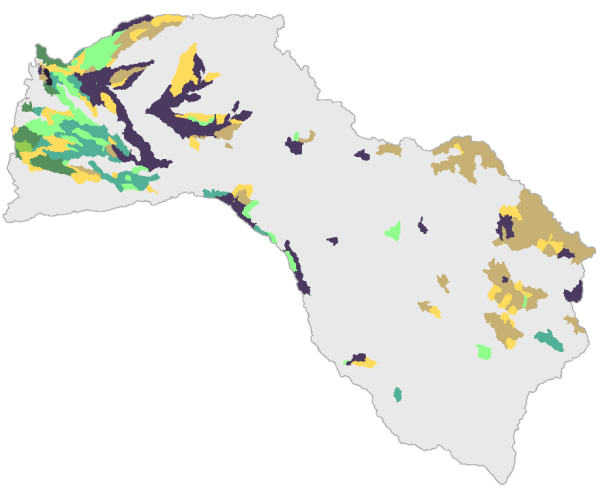

(c) $\beta_{1}=0.8, \beta_{2}=0$

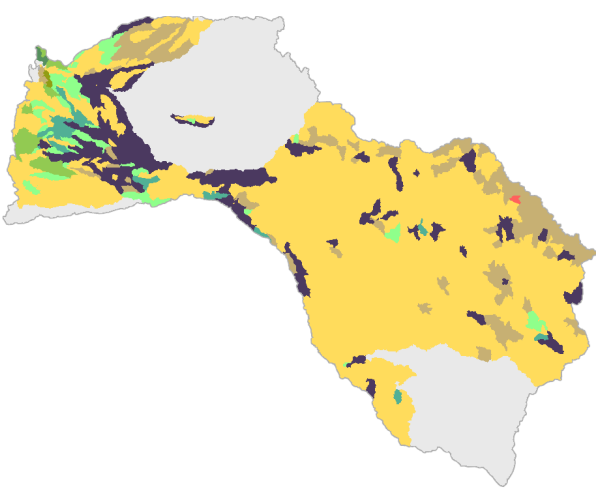

(e) $\beta_{1}=12, \beta_{2}=0$

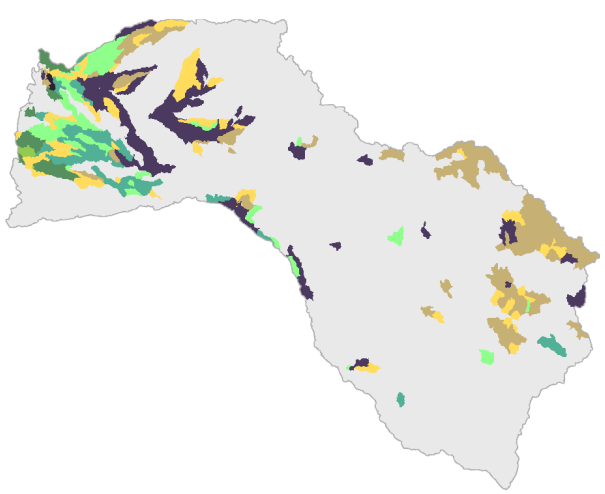

(b) $\beta_{1}=0.8, \beta_{2}=0$

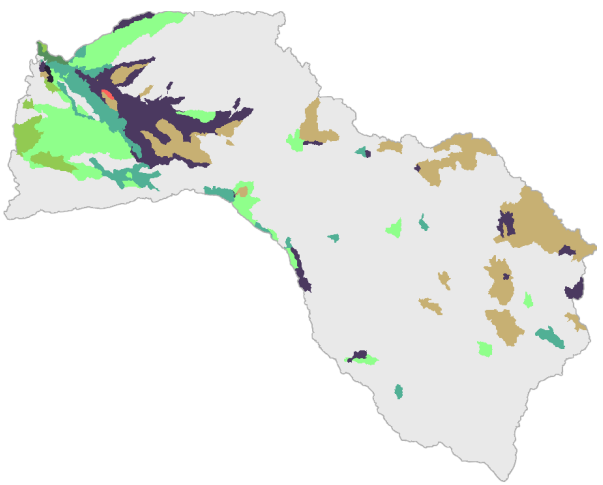

(d) $\beta_{1}=0.8, \beta_{2}=0.8$

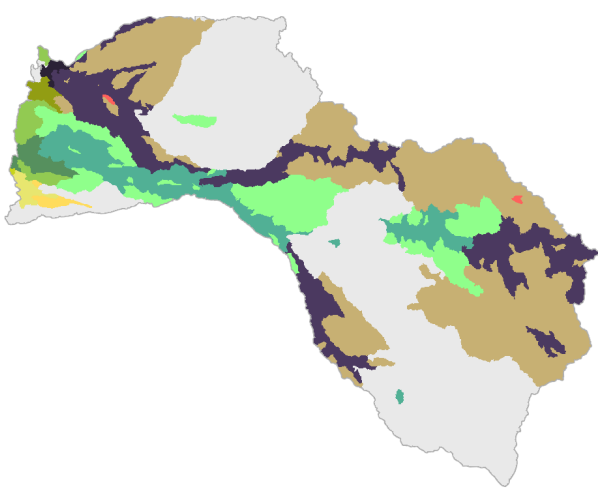

(f) $\beta_{1}=0.8, \beta_{2}=12$

\section{Actions}

\begin{tabular}{|l|l|}
\hline Monitoring \\
\hline Buffalo \\
\hline Toad \\
\hline Flow \\
Grazing \\
\hline Flow + Grazing \\
\hline
\end{tabular}

Toad+ Grazin Buffalo + Grazing Toad + Flow Buffalo + Flow Buffalo + Toad Toad + Flow + Grazing

Figure 4: Spatial distribution of actions selected by the MAMP and MAMP-E models. The management plans in (a), (c) and (e) were generated by different penalty factors by fragmentation of units $\left(\beta_{1}\right)$ while those in (b), (d) and (f) were generated by different penalty factors by fragmentation of actions $\left(\beta_{2}\right)$ 


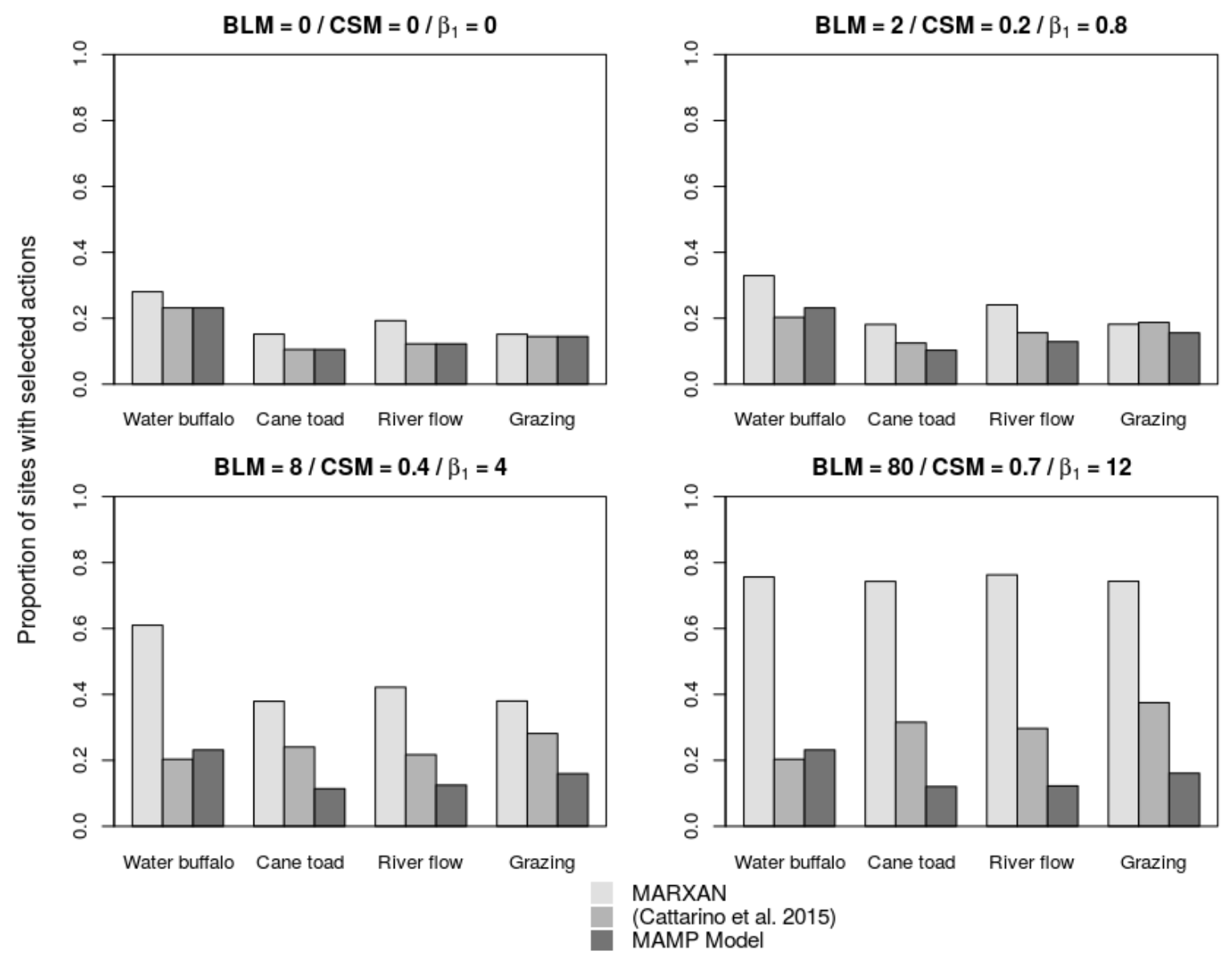

Figure 5: Portion of the units where actions are implemented, with respect to the total number of selected units, for different values of BLM (Boundary Length Modifier), CSM (Connectivity Strength Modifier) and $\beta_{1}$ (unit fragmentation penalty factor).

the proportions does not imply the selection of a significant number of new actions.

Regarding to the relationship between the average representation objective achieved across species $(\bar{B})$ and the respective costs of implementing the management plan, we observe the following. Using the MAMP model, there were no differences in the achievement of the average representation objective among species by increasing the penalty factor $\beta_{1}$, while with the extended version MAMP-E, increases in the penalty $\beta_{2}$ generated an increase in the average representation with approximately linear behavior (Figure 6. Regarding the behavior of the value of $\operatorname{cost} C$, the two curves show that in both cases this factor will increase if higher degrees of either type of connectivity are required. However, with MAMP at the highest penalty factor $\left(\beta_{1}=12\right)$, the cost ratio as defined as $\frac{C}{C_{T}}$ or $1-E$, is the same as for MAMP-E with penalty factor of $\beta_{1}=0.8$ and $\beta_{2}$ between 3.5 and 4 , but the ecological benefit of the extended model is $31.24 \%$ higher. 


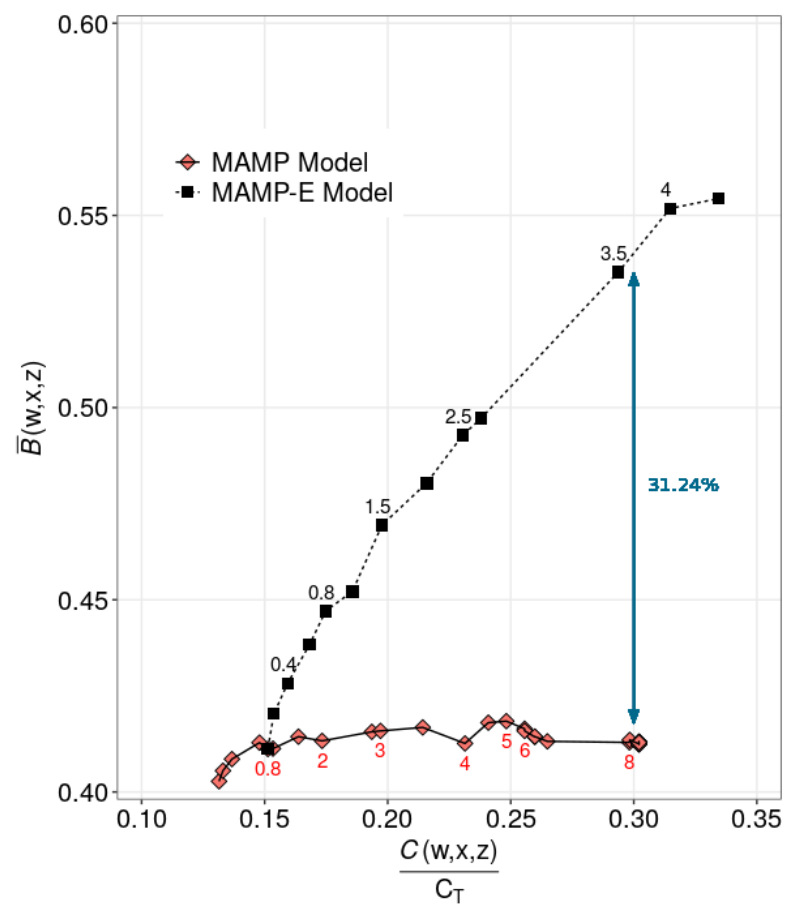

Figure 6: Level curves for the two proposed models showing the relationship between average benefit $(\bar{B})$ and the management plan cost ratio $\left(\frac{C}{C_{T}}\right)$ at different penalty factors (for MAMP, $\beta_{1}$ (below the points); for MAMP-E, $\beta_{2}$ with $\beta_{1}$ fixed at 0.8 (above the points)).

\section{Discussion}

Guidance on how to explicitly and efficiently prioritize the management of multiple threats, including deciding where to act, is a decision that has been studied by different authors [e.g. 20, 23, 34, 35], but to our knowledge, the proposed approach is the first to include mathematical programming to support these decisions. The use of an MIP approach provides a new framework to the problem of prioritization of the multiple actions, identifying efficient management plans to address multiple threats to the conservation of species and lays a basis for the development of more complex and realistic models to tackle a wide range of resource management problems. One of the advantages of our approach with respect to the already existing ones, is that it uses an exact method that allows to quantify the quality of the action plans, reaching in the case of study optimality gaps less than $1 \%$ for any level of spatial functionality, in running times less than 6 hours. Specifically, this MIP formulation has demonstrated three advantages over its heuristic (simulated annealing) counterparts: shorter execution times, higher quality solutions and a solution quality guarantee indicated by the gap percentage. This is aligned with the recommendations regarding the use of exact approaches over heuristics whenever possible as support for spatial conservation prioritization decisions [30]. Because of the flexibility of MIPbased approaches, it is a relatively simple matter to incorporate new constraints into the model, modify its objective and perform a sensitivity analysis on the parameters so that the effects of changes in management plans can be precisely 
measured. On the negative side, formulating a MIP problem often requires many variables and constraints, and as the resulting model grows in size, execution times may increase exponentially. But with advances in computer hardware, ever bigger problems can be handled and we may assume that in future, large instances will be increasingly manageable while heuristics will be able to find approximate solutions to complex problems, including non-linear ones, without great difficulty.

By identifying the specific actions needed to abate the threats affecting target species at each site, our MAMP model achieved higher efficiency in action management plans with respect to existing heuristic approaches when connectivity between selected sites was important. Our results align with those reported in [20]. This higher efficiency was reflected in a lower proportion of actions to be taken against three of the four threats studied, with water buffalo being the only ones that obtained greater proportions. This result may be due to a better balance between conservation actions, which, helped by the low presence of this threat in the basin, implies that its higher proportion does not considerably affect the efficiency of the management plans achieved. From the spatial point of view, when the connectivity requirement is zero, actions are guided by the distribution of scarce species that are below the threshold of the representation target, in addition to units with a high presence of species. While when the connectivity requirement increases, it implies the selection of sites only for monitoring, but no actions against threats are added in them. This is because MAMP only incorporates connectivity in units, i.e., once the target are achieved, additional units or sub-catchments are incorporated only to reduce fragmentation, with no further contribution to target achievement.

The selection of units with no actions against threats assigned, is not desirable because it might undermine the efficiency of solutions [23]. For this reason, accounting for connectivity within actions is not only a novel element but also an important one. As we report here, MAMP-E solutions, where connectivity within actions was accounted for, obtained up to $31.24 \%$ more average benefit $(\bar{B})$ using the same resources, compared to solutions when connectivity within the actions were not considered in MAMP. Differences in efficiency between MAMP and MAMP-E increased with the strength of connectivity within actions. We observed that MAMP-E outperforms MAMP when it comes to benefits for different cost levels, also obtaining a higher average degree of action connectivity $(\overline{D C A})$, and reaching degrees of unit connectivity (DCU) similar to those achieved by MAMP. This characteristic of MAMP-E is the result of grouping management actions rather than dispersing them over a wider area [22]; therefore, the model incorporates the generation of economies of scale. The incorporation of economies of scale through the connectivity of actions is an issue that had already been proposed by [36], but never directly demonstrated.

Although we have exposed the efficiency of our application approach in a freshwater context, the previous discussion demonstrates that we have developed a powerful approach to decision-making for the design and implementation of conservation management plans. So it can be applied to other biodiversity features and in different biogeographic contexts, 
where multiple threats affect multiple species in different ways. For example, it could be adapted for maritime, terrestrial environments, or the set of more than one them [37]. Likewise, the approach is flexible enough to incorporate the relative importance of the connectivity of actions and selected sites as part of the management plan, allowing it to be clear support for complex decision making.

\section{Conclusions}

We have developed a new methodological approach based on mathematical programming, which provides a more efficient framework to address the problem of prioritization of multiple conservation actions. Our approach is developed in a spatially explicit context that makes it suitable for solving complex problems involving spatial aspects of actions, and their impact on biodiversity. Comparing our MIP approach with the heuristic approach developed in [20] and Marxan, through a case study in Australia, an improvement in the quality of the solutions is demonstrated, as in the resolution time. In turn, we have incorporated a new expression of connectivity between actions, through the extended model (MAMP-E), which results in more efficient management plans regarding the model that only considers connectivity between the units (MAMP).

Regarding further development of the proposed models, a natural extension would be to incorporate the possibility of non-dichotomous responses by the targeted species to the implementation of a given conservation action. In other words, the actions would be modelled so as to consider varying probabilities of eradicating a given threat, rather than our simpler assumption that every implemented action eliminates its targeted threat completely. These further changes to the models could be taken a step further by considering continuous levels of the actual implementation of an action, with curves determining the species reactions at each level.

Acknowledgements J. Salgado-Rojas, E. Álvarez-Miranda and A. Weintraub acknowledge the support of the National Commission for Scientific and Technological Research CONICYT, Chile, through funding from DOCTORADO BECAS CHILE/2019 - 72200381, grants FONDECYT N.1180670, FONDECYT N.1191531 and through the Complex Engineering Systems Institute PIA/BASAL AFB180003. This research has received funding from the European Unions H2020 research and innovation program under the Marie Sklodowska-Curie grant agreement No. 691149 (SuFoRun). V. Hermoso and J. Garcia-Gonzalo acknowledge the support from MINECO (RYC-2013-14262 and RYC-2013-13979) and from CERCA Programme/Generalitat de Catalunya. 


\section{References}

[1] S. Díaz, J. Settele, E. Brondízio, H. Ngo, M. Guèze, J. Agard, A. Arneth, P. Balvanera, K. Brauman, S. Butchart, et al., Summary for policymakers of the global assessment report on biodiversity and ecosystem services of the Intergovernmental Science-Policy Platform on Biodiversity and Ecosystem Services (2019).

[2] P. Leadley, C. Krug, R. Alkemade, H. Pereira, U. Sumaila, M. Walpole, A. Marques, T. Newbold, L. Teh, J. van Kolck, Progress towards the Aichi Biodiversity Targets: An assessment of biodiversity trends, policy scenarios and key actions, Secretariat of the Convention on Biological Diversity (2017).

[3] WWF. 2018. Living Planet Report - 2018: Aiming Higher. Grooten, M. and Almond, R.E.A.(Eds). WWF, Gland, Switzerland.

[4] M. Groom, G. Meffe, C. Carroll, S. Andelman, Principles of conservation biology, no. Sirsi) i9780878935185, Sinauer Associates Sunderland, 2006.

[5] Y. Wiersma, D. Sleep, A review of applications of the six-step method of systematic conservation planning, The Forestry Chronicle 92 (3) (2016) 322-335.

[6] J. Williams, C. ReVelle, S. Levin, Spatial attributes and reserve design models: a review, Environmental Modeling \& Assessment 10 (3) (2005) $163-181$.

[7] A. Moilanen, J. Leathwick, J. Quinn, Spatial prioritization of conservation management, Conservation Letters 4 (5) (2011) $383-393$.

[8] V. Hermoso, S. Januchowski-Hartley, S. Linke, Systematic planning of disconnection to enhance conservation success in a modified world, Science of the Total Environment 536 (2015) 1038-1044.

[9] J. Liang, X. He, G. Zeng, M. Zhong, X. Gao, X. Li, X. Li, H. Wu, C. Feng, W. Xing, et al., Integrating priority areas and ecological corridors into national network for conservation planning in china, Science of The Total Environment 626 (2018) $22-29$.

[10] K. Jones, J. Watson, H. Possingham, C. Klein, Incorporating climate change into spatial conservation prioritisation: A review, Biological Conservation 194 (2016) 121-130.

[11] C. Wang, J. Wan, Z. Zhang, L. Zhao, et al., Integrating climate change into conservation planning for taxuschinensis, an endangered endemic tree plant in china., JAPS: Journal of Animal \& Plant Sciences 27 (1).

[12] C. Runge, A. Tulloch, H. Possingham, V. Tulloch, R. Fuller, Incorporating dynamic distributions into spatial prioritization, Diversity and Distributions 22 (3) (2016) 332-343.

[13] J. Diamond, The island dilemma: lessons of modern biogeographic studies for the design of natural reserves, Biological Conservation 7 (2) (1975) 129-146.

[14] J. Williams, C. ReVelle, S. Levin, Using mathematical optimization models to design nature reserves, Frontiers in Ecology and the Environment 2 (2) (2004) 98-105.

[15] M. Watts, I. Ball, R. Stewart, C. Klein, K. Wilson, C. Steinback, R. Lourival, L. Kircher, H. Possingham, Marxan with zones: Software for optimal conservation based land-and sea-use zoning, Environmental Modelling \& Software 24 (12) (2009) 1513-1521.

[16] H. Possingham, J. Day, M. Goldfinch, F. Salzborn, The mathematics of designing a network of protected areas for conservation, in: Decision Sciences: Tools for Today. Proceedings of 12th National ASOR Conference, 1993, pp. 536-545.

[17] J. Lehtomäki, A. Moilanen, Methods and workflow for spatial conservation prioritization using zonation, Environmental Modelling \& Software 47 (2013) 128-137.

[18] E. Game, P. Kareiva, H. Possingham, Six common mistakes in conservation priority setting, Conservation Biology 27 (3) (2013) $480-485$.

[19] J. Carwardine, T. OConnor, S. Legge, B. Mackey, H. Possingham, T. Martin, Prioritizing threat management for biodiversity conservation, Conservation Letters 5 (3) (2012) 196-204.

[20] L. Cattarino, V. Hermoso, J. Carwardine, M. Kennard, S. Linke, Multi-action planning for threat management: a novel approach for the spatial prioritization of conservation actions, PLOS one 10 (5) (2015) e0128027.

[21] K. Wilson, E. Underwood, S. Morrison, K. Klausmeyer, W. Murdoch, B. Reyers, G. Wardell-Johnson, P. Marquet, P. Rundel, M. McBride, et al., Conserving biodiversity efficiently: what to do, where, and when, PLOS biology 5 (9) (2007) e223.

[22] N. Auerbach, A. Tulloch, H. Possingham, Informed actions: where to cost effectively manage multiple threats to species to maximize return on investment, Ecological Applications 24 (6) (2014) 1357-1373.

[23] L. Cattarino, V. Hermoso, L. Bradford, J. Carwardine, K. Wilson, M. Kennard, S. Linke, Accounting for continuous species' responses to management effort enhances cost-effectiveness of conservation decisions, Biological conservation 197 (2016) 116-123. 
[24] A. Moilanen, Methods for reserve selection: interior point search, Biological Conservation 124 (4) (2005) $485-492$.

[25] J. Kirkpatrick, An iterative method for establishing priorities for the selection of nature reserves: an example from tasmania, Biological Conservation 25 (2) (1983) 127-134.

[26] K. Cocks, I. Baird, Using mathematical programming to address the multiple reserve selection problem: an example from the eyre peninsula, south australia, Biological Conservation 49 (2) (1989) 113-130.

[27] S. Sarkar, Complementarity and the selection of nature reserves: Algorithms and the origins of conservation planning, 1980-1995, Archive for History of Exact Sciences 66 (4) (2012) 397-426.

[28] A. Billionnet, Mathematical optimization ideas for biodiversity conservation, European Journal of Operational Research 231 (3) (2013) 514-534.

[29] H. Önal, Y. Wang, S. Dissanayake, J. Westervelt, Optimal design of compact and functionally contiguous conservation management areas, European Journal of Operational Research 251 (3) (2016) 957-968.

[30] H. Beyer, Y. Dujardin, M. Watts, H. Possingham, Solving conservation planning problems with integer linear programming, Ecological Modelling 328 (2016) 14-22.

[31] V. Hermoso, S. Linke, J. Prenda, H. Possingham, Addressing longitudinal connectivity in the systematic conservation planning of fresh waters, Freshwater Biology 56 (1) (2011) 57-70.

[32] M. Beger, S. Linke, M. Watts, E. Game, E. Treml, I. Ball, H. Possingham, Incorporating asymmetric connectivity into spatial decision making for conservation, Conservation Letters 3 (5) (2010) 359-368.

[33] J. Ardron, H. Possingham, C. Klein, Marxan good practices handbook, Pacific Marine Analysis and Research Association, Vancouver (2008) 149.

[34] N. Auerbach, K. Wilson, A. Tulloch, J. Rhodes, J. O. Hanson, H. Possingham, Effects of threat management interactions on conservation priorities, Conservation Biology 29 (6) (2015) 1626-1635.

[35] C. Mantyka-Pringle, T. Martin, D. Moffatt, J. Udy, J. Olley, N. Saxton, F. Sheldon, S. Bunn, J. Rhodes, Prioritizing management actions for the conservation of freshwater biodiversity under changing climate and land-cover, Biological Conservation 197 (2016) 80-89.

[36] L. Cattarino, V. Hermoso, J. Carwardine, V. Adams, M. Kennard, S. Linke, Information uncertainty influences conservation outcomes when prioritizing multi-action management efforts, Journal of applied ecology 55 (5) (2018) 2171-2180.

[37] V. Adams, J. Álvarez-Romero, J. Carwardine, L. Cattarino, V. Hermoso, M. Kennard, S. Linke, R. Pressey, N. Stoeckl, Planning across freshwater and terrestrial realms: cobenefits and tradeoffs between conservation actions, Conservation Letters 7 (5) (2014) 425-440. 